\title{
New insights into the phenotypic spectrum of 14q22q23 deletions: a case report and literature review
}

\author{
Anna Pichiecchio ${ }^{1,2}$, Giovanni Vitale $2^{2^{*}}$ (D), Camilla Caporali ${ }^{2}$, Cecilia Parazzini ${ }^{3}$, Donatella Milani ${ }^{4}$, \\ Maria Paola Recalcati ${ }^{5}$, Laura D'Amico ${ }^{2}$, Sabrina Signorini', Umberto Balottin ${ }^{2,6}$ and Stefano Bastianello ${ }^{1,2}$
}

\begin{abstract}
Background: Mutations occurring in the orthodenticle homeobox 2 gene (OTX2) are responsible for a rare genetic syndrome, characterized mainly by microphthalmia/anophthalmia associated with extra-ocular defects such as brain malformations, pituitary abnormalities, short stature and intellectual disability. To date, the spectrum of radiological features observed in patients with OTX2 mutations has never been summarized.

Case presentation: In this report, we describe a case of large microdeletion encompassing OTX2 but not BMP4 presenting with a syndromic anophthalmia with corpus callosum hypoplasia, pituitary gland hypoplasia and vermian hypoplasia.

Conclusion: Our case report provides an illustration of the neuroradiological spectrum in a case of OTX2-related syndrome and the first radiological evidence of 14q22.2q23.1 deletion associated posterior cranial fossa anomalies.
\end{abstract}

Keywords: OTX2, MRI, Microphthalmia, Anophthalmia, Pituitary, Cerebellum

\section{Background}

The orthodenticle homeobox 2 gene (OTX2, OMIM \#600037) encodes a member of the bicoid subfamily of homeodomain-containing transcription factors, and it plays a crucial role in brain, pituitary gland, sensory organ and craniofacial development. More specifically, it is involved in several processes, which include: forebrain induction and specification, pituitary and GnRH neuronal system development eye formation (playing a major role in retinal pigment epithelium specification) and migration of neural crest cells from the hindbrain (which leads to the development of the maxillary and mandibular prominences) [1]. Furthermore, in the developing brain of the mouse embryo, it influences the activity of the isthmic organizer (midbrain-hindbrain boundary) through its expression in the rostral-medial ends of the cerebellar primordia (vermis-forming epithelium) [1].

Mutations in OTX2 exhibit incomplete penetrance and broad extra and intrafamilial phenotypic variability $[2,3]$.

* Correspondence: giovitale01@gmail.com

${ }^{2}$ University of Pavia, Corso Strada Nuova 65, 27100 Pavia, Italy

Full list of author information is available at the end of the article
The major phenotype reported in patients with OTX2 mutations consists of isolated or syndromic microphthalmia/anophthalmia, possibly associated with extra-ocular defects such as brain malformations, pituitary abnormalities, short stature and intellectual disability [4]. Three distinct syndromic diseases are linked to haploinsufficiency of OTX2, namely combined pituitary hormone deficiency 6 (CPHD6, OMIM \#613986), syndromic microphthalmia 5 (MCOPS5, OMIM \#610125) and otocephaly/agnathia complex [5]. OTX2 mutations are the second most common genetic cause of microphthalmia/anophthalmia (after SOX2); furthermore, the gene is responsible for a very small proportion (less than 1\%) of infantile retinal disorders, such as Leber's congenital amaurosis [1].

The pathogenic effect is probably due to an haploinsufficiency mechanism; some cases of microdeletions encompassing OTX2 are reported: anophthalmia/microphthalmia, other ocular defects, pituitary disfunction, anomalies of the extremities, cardiac malformations, urogenital abnormalities, are described. Regarding the

(c) The Author(s). 2018 Open Access This article is distributed under the terms of the Creative Commons Attribution 4.0 International License (http://creativecommons.org/licenses/by/4.0/), which permits unrestricted use, distribution, and reproduction in any medium, provided you give appropriate credit to the original author(s) and the source, provide a link to the Creative Commons license, and indicate if changes were made. The Creative Commons Public Domain Dedication waiver (http://creativecommons.org/publicdomain/zero/1.0/) applies to the data made available in this article, unless otherwise stated. 
extraocular involvement, the phenotypic spectrum of OTX2 mutations included structural and functional abnormalities of the pituitary gland, global developmental delay, autism, attention-deficit disorder, feeding difficulties, seizures and microcephaly other structural brain anomalies, affecting the corpus callosum and hippocampus with no clear genotype-phenotype correlations [6-9]. Also large deletions encompassing OTX2 involving also $B M P 4$ were previously described associated with syndromic anophtalmia phenotype including microcephaly, sensorineural deafness, abnormalities of extremities, cryptorchidism, partial callosal agenesis, cerebellar and pituitary abnormalities, and developmental delay [10].

To the best of our knowledge, we here report the first case of posterior fossa involvement in a patient with a microdeletion encompassing the OTX2 gene, and also review the radiological findings described in literature reports of $O T X 2$ mutations and deletions.

\section{Case presentation}

The patient here described is the first child of Caucasian healthy non-consanguineous parents, born at the 35th week of gestation by natural delivery following premature rupture of the membranes. The pregnancy was otherwise unremarkable. He has an older maternal half-sister, and his mother previously suffered a miscarriage at the 6th week of gestation.
At birth, the child presented with enophthalmia with right blepharophimosis, cryptorchidism and scrotal hypoplasia; auxological parameters were normal. Echocardiography and a complete abdomen ultrasound examination gave normal findings. Brain and orbital magnetic resonance imaging (MRI) (Fig. 1) showed a complex set of malformations: right microphthalmia and homolateral agenesis of the optic nerve and hemi-chiasm, a small posterior fossa with more vertical and caudal tentorial implant, and a wider-than-normal IV ventricle due to cerebellar vermis hypoplasia. The pituitary gland was normal. Blood samples were taken for array-CGH analysis (patient and parents) and molecular analysis of the microphthalmia-associated genes (SOX2, GDF6, PAX6, SHH, RAX, OTX, VSX2). The array-CGH analysis was performed according to standard protocols, using an oligonucleotide array with an average resolution of 130 $\mathrm{Kb}$. The analysis showed a de novo 6,41 Mb deletion at 14q22.2-q23.1 (55386907-61,795,829, NCBI Build 37 hg19, February 2009), involving 43 genes including OTX2 and other 7 genes reported as disease causing in OMIM database (Fig. 2). Molecular analysis revealed the genomic variant c.1271C > T (p.Pro424Leu) in the $S H H$ gene, in heterozygosis. To date, this variant, maternally inherited, lacks clear pathogenic significance: since a genetic cause of microphthalmy had already been found and considering the mother showed no clinical or neuroradiological signs, the laboratory signaled it as probably not pathogenic.

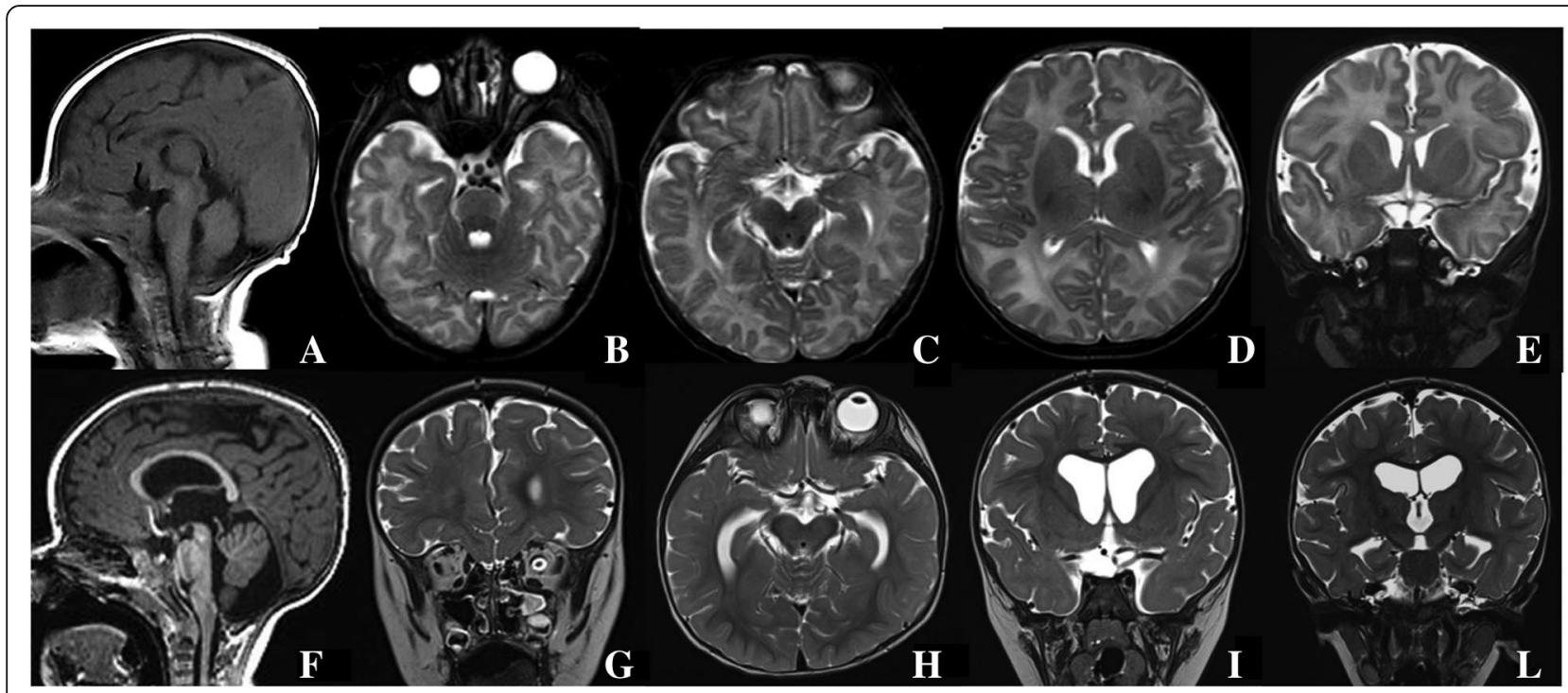

Fig. 1 Brain MRI at birth and at the age of 18 months. Brain MRI at birth (a-e): sagittal T1-weighted spin echo (SE) (a), axial T2-weighted turbo spin echo (TSE) (b-d), coronal T2-weighted TSE (e). The examination shows right microphthalmia (b) and agenesis of the right optic nerve and hemi-chiasm (a-c), normal pituitary gland and stalk (a and e), small cranial posterior fossa with vertical and caudal tentorial implant, and a widerthan-normal IV ventricle due to cerebellar vermis hypoplasia (a). No molar tooth sign is evident at the midbrain level (c). Follow-up brain MRI at the age of 18 months (e-l): sagittal T1-weighted SE (e), coronal T2-weighted TSE (f, i, l) and axial T2-weighted TSE (h). The examination confirms the eyeball, optic nerve and posterior fossa findings, and clearly displays slight vermian dysmorphism and a wide communication between the IV ventricle and the basal cisterns (f), with regular superior cerebellar peduncles (f), corpus callosum hypoplasia (f), ventricular enlargement (i and $\mathbf{I})$, incomplete hippocampal inversion (I) and pituitary gland hypoplasia (f and $\mathbf{i}$ ) 


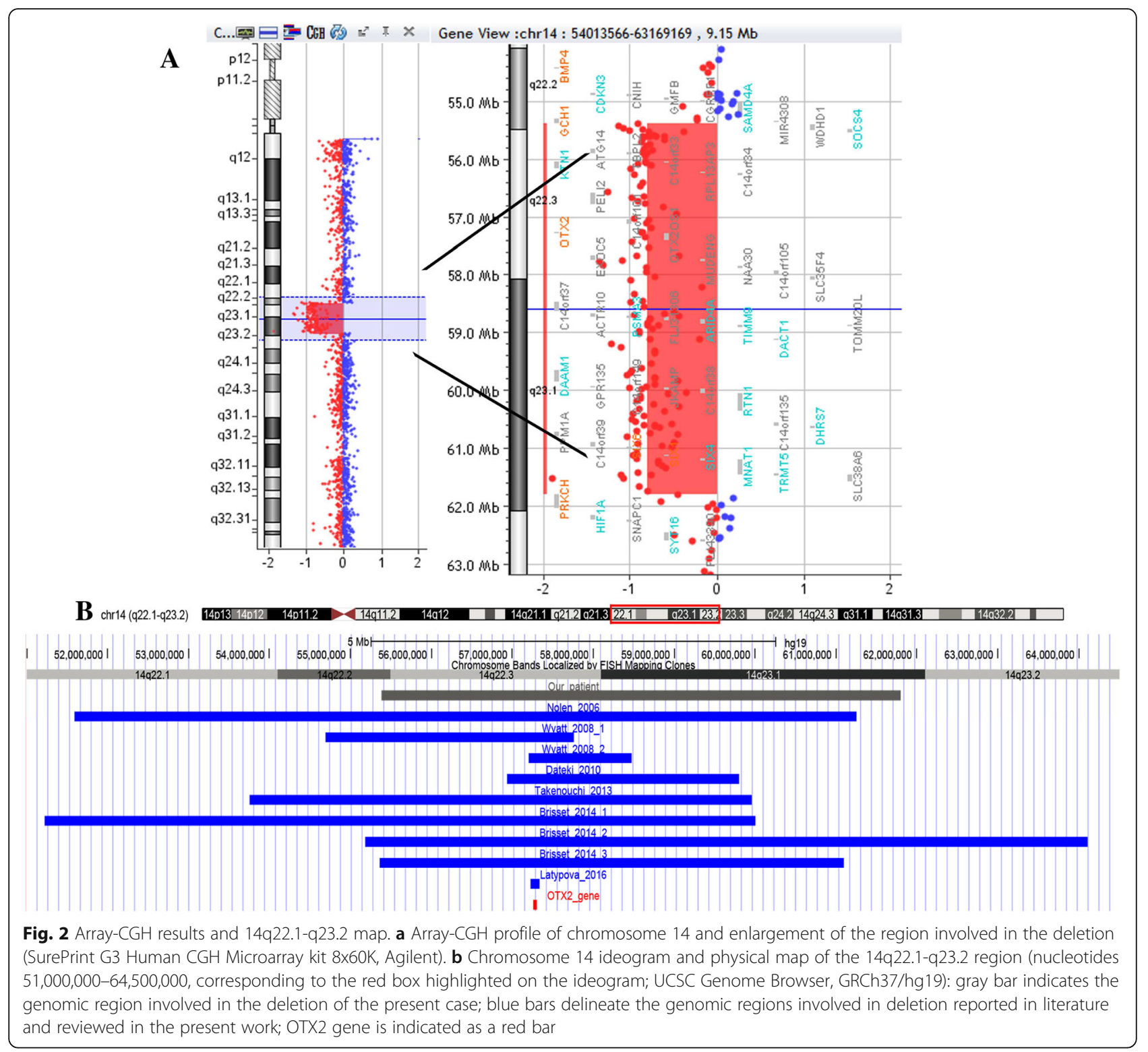

The child underwent right eye enucleation and replacement with an ocular prosthesis. At 2 months of age, left choanal atresia was diagnosed by nasal endoscopy performed due to breathing difficulties. By the age of 9 months he was showing severe growth retardation without evident deficiency of pituitary hormones. Neurological examination revealed generalized hypotonia with preserved reflexes. He presented exodeviation and erratic movements of the left eye. Visual localization was possible only for multisensory targets placed at close range, and horizontal smooth pursuit could not be evoked without head adjustments. Visual evoked potentials showed increased latency, while electroretinography was unreliable due to the child's uncooperativeness. No seizures were reported but EEG recording indicated poor spatiotemporal organization and generalized waveform abnormalities during sleep. At the age of 18 months, he showed severe developmental delay in terms of acquisition of the main communicative and motor milestones. He also displayed stereotypic behaviors, but was able to interact with his parents, smiling spontaneously in response to their voices and exploring their faces with his hands. Follow-up brain MRI (Fig. 1) confirmed the eyeball, optic nerve and posterior fossa findings, and revealed slight vermian dysmorphism, corpus callosum hypoplasia, a thin anterior commissure, global hemispheric white matter reduction, ventricular enlargement, a wide communication between the IV ventricle and the basal cisterns, dysmorphism of the temporal horns of the lateral ventricles due to incomplete hippocampal inversion, and 
pituitary gland hypoplasia $\left(101.5 \mathrm{~mm}^{3}\right)$ [11]. Pituitary hormone levels were still within the normal range, but an endocrinological follow-up remains mandatory due to the young age of the child, his severe growth retardation and the presence, from the birth, of scrotal hypoplasia.

\section{Discussion and conclusions}

Mutations, including deletions, in OTX2 are responsible for a broad spectrum of morphological abnormalities, associated with high phenotypic heterogeneity, proportional to the numerous pathways of cell differentiation and migration in which the gene is involved [1].

Brain MRI makes it possible to establish the severity of several clinically evident malformations, highlighting orbital and cerebral abnormalities that can be further subdivided into: eyeball and visual pathway dysgenesis/ agenesis, pituitary malformations, and brain malformations. Table 1 summarizes the radiological findings described in available literature reports of OTX2 mutations; these are schematically represented in Fig. 3.

OTX2 first expression starts within the optic vescicle, then it becomes specifically restricted to retinal pigment epithelium territory and later on is also found in the neural retina [12]. Eye development depends on the number of functional copies of Otx, especially of OTX2; embryos carrying the minimum Otx dosage compatible with viability show gross eye malformations such as microphthalmia or anophthalmia and agenesis of the lens. As consequence also the optic nerve, which is composed of retinal ganglion cell axons and supporting glial cells, could be affected in OTX2 mutations in the form of optic nerve hypoplasia [13].With regard to eyeball and visual pathway dysgenesis/agenesis, MRI has been shown to allow optimal characterization of microphthalmia/anophthalmia (both monolateral and bilateral), and it can reveal the presence, albeit rare, of orbital cystic remnants. Furthermore, even though OTX2 is expressed in the optic nerve sheath, but not in the optic nerve itself, cases of optic nerve and chiasmatic hypoplasia/aplasia, as found in our patient, have been described; this finding is probably due to retrograde trans-synaptic degeneration. Interestingly, anophthalmia and microphthalmia can both be associated with optic nerve aplasia or hypoplasia.

OTX2 mutations are also associated with variable hormonal-morphological pituitary phenotypes $[1,14]$. $\mathrm{GH}$ is the most vulnerable pituitary hormone in OTX2 mutations, and it can be deficient even when the gland appears normal, possibly because the gene is also involved in regulating the secretion of GnRH by the hypothalamus [14]. However, pituitary dysfunction is more commonly reported in association with developmental abnormalities of the gland, specifically anterior lobe aplasia/hypoplasia (with altered saddle conformation), ectopic/absent posterior lobe, and an invisible or interrupted stalk [14]. Our case was found to have normal pituitary function, despite showing hypoplasia of the gland, a finding which became more evident at 18 months, still in the absence of related hormonal disorders. This suggests that an MRI re-evaluation, also with 3D acquisition in doubtful cases, could provide the clinician with additional information, and therefore that the decision on whether or not it is warranted should be made independently of hormonal abnormalities.

Brain malformations in OTX2 mutation include ventricular dilatation, partial corpus callosum agenesis, and reduced hemispheric white matter [1]. Hippocampal abnormal gyration has been described in two patients; interestingly, the hippocampus originates from the alar plate, which develops from an OTX2-expressing domain of the neural plate [1]. In our case we documented all these radiological findings, in particular global ventricular enlargement, diffuse white matter reduction with normal myelination, incomplete hippocampal inversion leading to dysmorphism of the temporal horns, and corpus callosum hypoplasia with a thin anterior commissure.

Moreover, our case also showed a verticalized tentorial implant bordering a small posterior fossa, and a hypoplastic and slightly dysmorphic vermis. To date, literature descriptions of malformations of the posterior cranial fossa, due to a large microdeletion encompassing both BMP4 and OTX2, consist of an old report of an autopsy finding of cerebellar hypoplasia in a fetus [15] and a more recent description of Chiari malformation [10]. The latter fails to specify the type of Chiari malformation, while the image provided deals with the pituitary findings. Moreover, two cases of vermian hypoplasia have been described, but in the presence of a concurrent OTX2-BMP4 deletion [10]. A case of vermian heterotopia and brain cortical dysplasia has been reported, but other genetic mechanisms related to cortical development malformations were not excluded [16]. Althogh we cannot rule out a specific role of OTX2 haploinsufficiency in vermis hypoplasia, vermian involvement in cases with OTX2 mutations could not be surprising, as it is consistent what is known about OTX2 activity in cerebellar development. In fact, OTX2 is expressed in the rostral-medial ends of the cerebellar primordia of the mouse embryo (the vermis-forming epithelium), suggesting that it plays a role in local neurogenesis. In support of the significance of OTX2 in human cerebellar development, it has been demonstrated that OTX2 acts as a repressor of myogenic and neuronal differentiation in medulloblastoma cells [1]. 


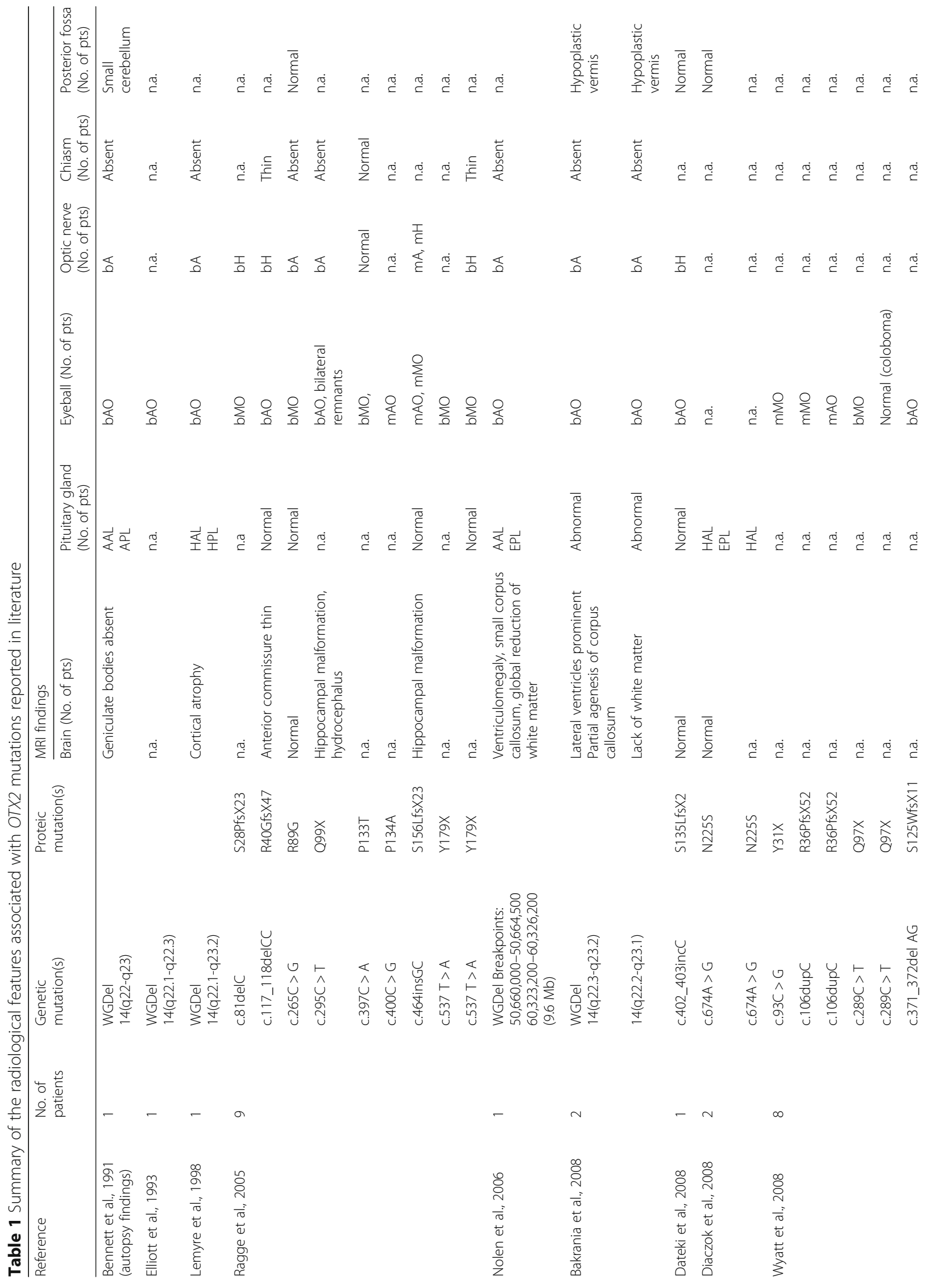




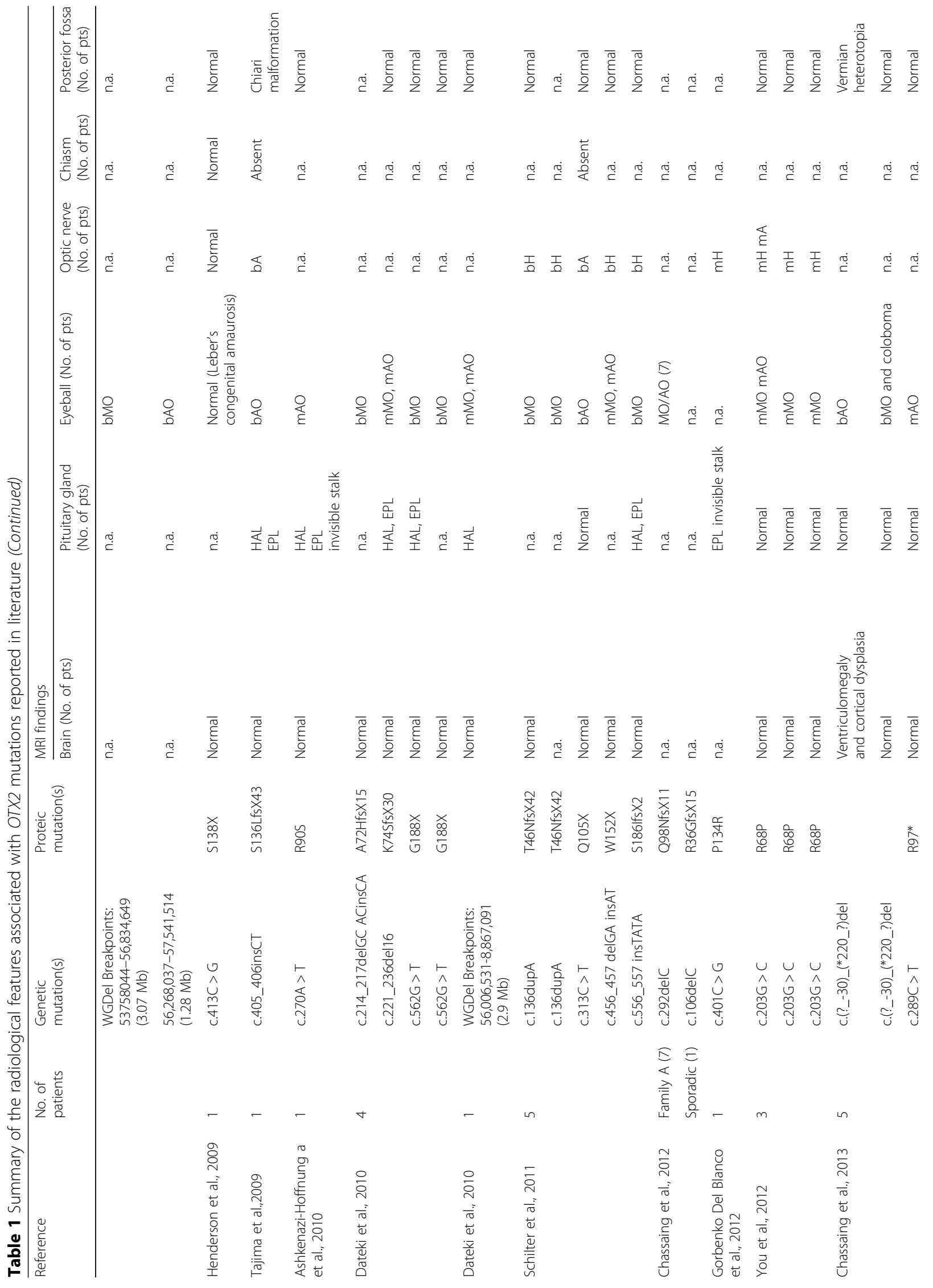




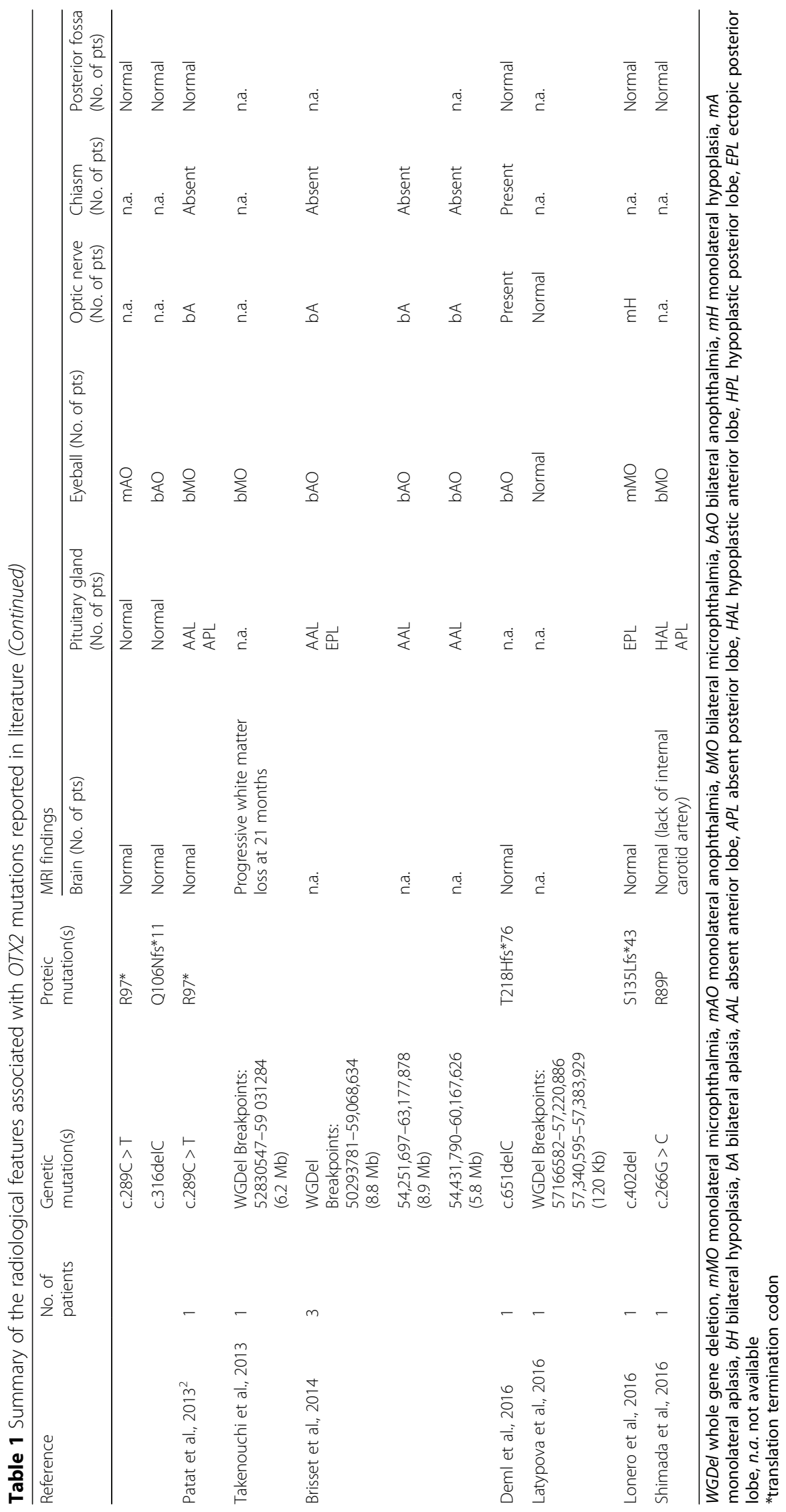




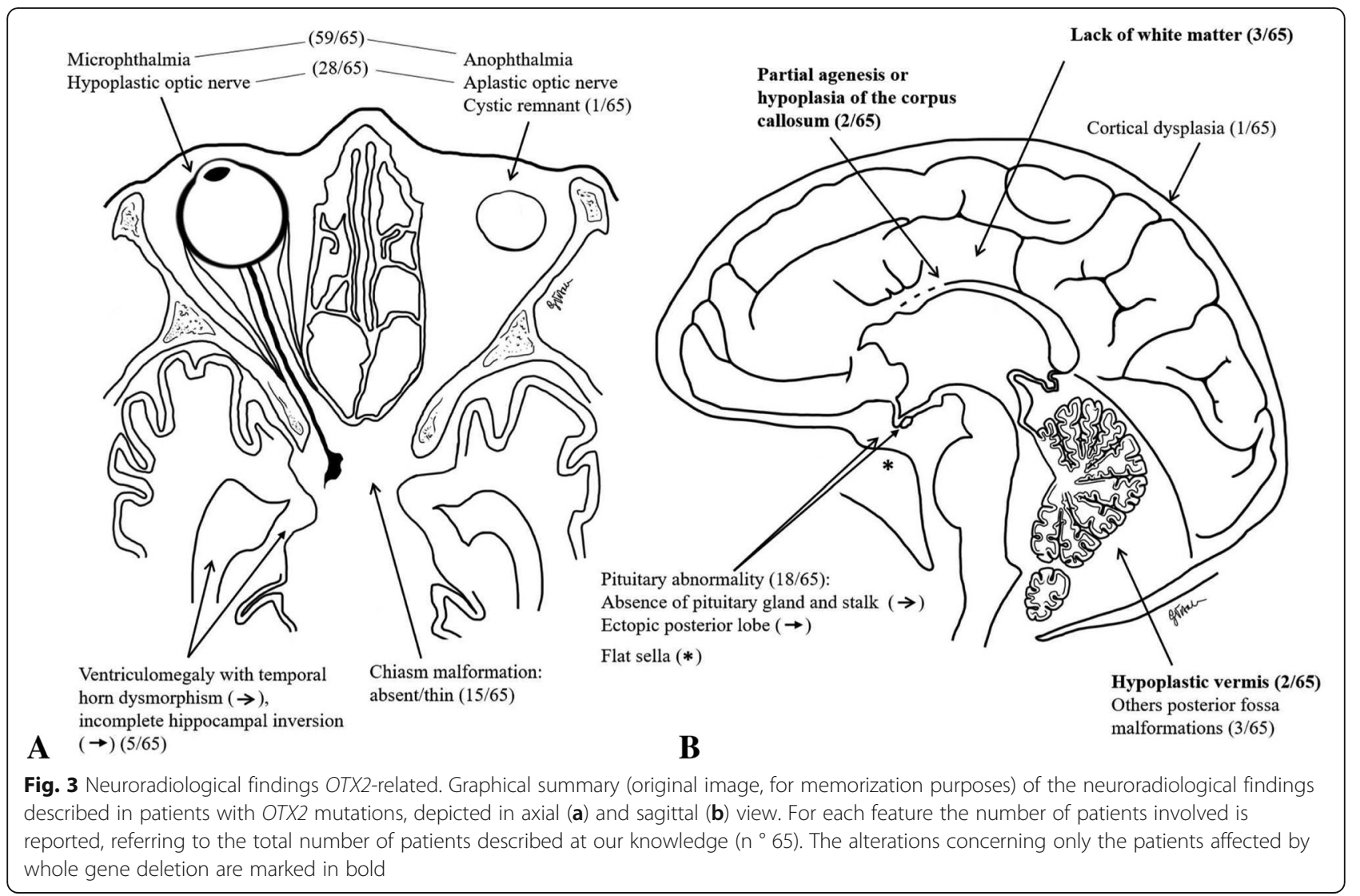

Microdeletions involving OTX2 are not classically associated with cerebellar malformations. In our case, more than 20 of the genes involved in the microdeletion are expressed in cerebellum, but only three are associated with human diseases. In particular, TMEM260 and TRMT5 are associated with recessive diseases without cerebellar involvement, while KIAA0586 is associated with Joubert syndrome 23 (JBTS23, MIM 616490).

Eventually, regarding phenotypic features, choanal atresia could be a misleading finding in our case report, leading the clinician to consider firstly CHARGE syndrome, due to deletion/duplication of CHD7 or SPINT2 mutations, another gene associated with developmental eye defects and choanal atresia as well as gut abnormalities. As a limit of our study, whole exome sequencing could not be performed, neither deletion/duplication analysis of $\mathrm{CHD} 7$ or potential coincident recessive pathogenic variants in SPINT2, but choanal atresia has been reported as associated to OTX2 mutations $[8,17]$. Moreover ocular malformation of our patient characterized only by right microphthalmia, was not associated with common features of CHARGE syndrome such as coloboma, heart defects, genitourinary anomalies, ear anomalies and facial dimorphisms or with gut abnormalities typical of SPINT2 mutations.
In conclusion, our case report provides an illustration of the neuroradiological spectrum that characterizes patients with OTX2-related syndrome, defined by microphthalmia/anophthalmia associated with extra-ocular defects such as brain malformations, pituitary abnormalities, short stature and intellectual disability. It also provides the first radiological evidence of OTX2 deletion with associated posterior cranial fossa anomalies.

\section{Acknowledgements}

We thank Dr. Catherin Wrenn for english revision.

Funding

This study was supported by grant of the Italian Ministry of Health "RC2017".

Availability of data and materials

Data sharing not applicable to this article as no datasets were generated or analysed during the current study.

\section{Authors' contributions}

AP wrote the manuscript, analyzed and interpreted radiological data; GV wrote the manuscript, reviewed the literature; CC analyzed and interpreted clinical data; CP wrote the manuscript, analyzed and interpreted radiological data; DM wrote the manuscript, analyzed and interpreted genetic data; MPR analyzed and interpreted array-CGH test; LD analyzed and interpreted clinical data; SS wrote the manuscript, analyzed and interpreted clinical data; UB made the final revision; SB made the final revision. All authors read and approved the final manuscript. 
Ethics approval and consent to participate

Not applicable.

\section{Consent for publication}

Written informed consent was obtained from the patient's parents for publication of patient's medical data and images.

\section{Competing interests}

The authors declare that they have no competing interests.

\section{Publisher's Note}

Springer Nature remains neutral with regard to jurisdictional claims in published maps and institutional affiliations.

\section{Author details}

'Department of Neuroradiology, IRCCS Mondino Foundation, via Mondino 2, 27100 Pavia, Italy. ${ }^{2}$ University of Pavia, Corso Strada Nuova 65, 27100 Pavia, Italy. ${ }^{3}$ Department of Pediatric Radiology and Neuroradiology, Children's Hospital "V. Buzzi", via Lodovico Castelvetro 32, 20154 Milan, Italy. ${ }^{4}$ Medical Genetics Unit, Fondazione IRCCS Ca' Granda Ospedale Maggiore Policlinico, via Francesco Sforza 35, 20122 Milan, Italy. ${ }^{5}$ Medical Cytogenetics Laboratory, IRCCS Istituto Auxologico Italiano, Milan, Italy. ${ }^{6}$ Child Neuropsychiatry Unit, IRCCS Mondino Foundation, via Mondino 2, 27100 Pavia, Italy.

Received: 28 August 2018 Accepted: 20 September 2018 Published online: 29 September 2018

\section{References}

1. Beby F, Lamonerie T. The homeobox gene Otx2 in development and disease. Exp Eye Res. 2013;111:9-16. https://doi.org/10.1016/j.exer.2013. 03.007.

2. Ragge NK, Brown AG, Poloschek CM, et al. Heterozygous mutations of OTX2 cause severe ocular malformations. Am J Hum Genet. 2005;76:1008-22. https://doi.org/10.1086/430721.

3. Williamson KA, FitzPatrick DR. The genetic architecture of microphthalmia, anophthalmia and coloboma. Eur J Med Genet. 2014;57(8):369-80. https:// doi.org/10.1016/.j.jmg.2014.05.002.

4. Schilter KF, Schneider A, Bardakjian T, et al. OTX2 microphthalmia syndrome: four novel mutations and delineation of a phenotype. Clin Genet. 2011;79: 158-68. https://doi.org/10.1111/j.1399-0004.2010.01450.x.

5. Chassaing N, Sorrentino S, Davis EE, et al. OTX2 mutations contribute to the otocephalydysgnathia complex. J Med Genet. 2012;49(6):373-9. https://doi. org/10.1136/jmedgenet-2012-100892.

6. Nolen LD, Amor D, Haywood A, et al. Deletion at 14q22-23 indicates a contiguous gene syndrome comprising anophthalmia, pituitary hypoplasia, and ear anomalies. Am J Med Genet A. 2006;140(16):1711-8. https://doi.org/ 10.1002/ajmg.a.31335.

7. Ashkenazi-Hoffnung $L$, Lebenthal $Y$, Wyatt AW, et al. A novel loss-of-function mutation in OTX2 in a patient with anophthalmia and isolated growth hormone deficiency. Hum Genet. 2010 Jun;127(6):721-9. https://doi.org/10, 1007/s00439-010-0820-9.

8. Martínez-Frías ML, Ocejo-Vinyals JG, Arteaga R, et al. Interstitial deletion 14q22.3-q23.2: Genotype-phenotype correlation. Am J Med Genet A. 2014; 164A(3):639-47. https://doi.org/10.1002/ajmg.a.36330.

9. Wyatt A, Bakrania P, Bunyan DJ, et al. Novel heterozygous OTX2 mutations and whole gene deletions in anophthalmia, microphthalmia and coloboma. Hum Mutat. 2008;29:E278-83. https://doi.org/10.1002/humu.20869.

10. Bakrania P, Efthymiou M, Klein JC, et al. Mutations in BMP4 cause eye, brain, and digit developmental anomalies: overlap between the BMP4 and hedgehog signaling pathways. Am J Hum Genet. 2008;82(2):304-19. https:// doi.org/10.1016/j.ajhg.2007.09.023.

11. Fink AM, Vidmar S, Kumbla S, et al. Age-related pituitary volumes in prepubertal children with normal endocrine function: volumetric magnetic resonance data. J Clin Endocrinol Metab. 2005;90(6):3274-8. https://doi.org/ 10.1210/jc.2004-1558.

12. Bovolenta $\mathrm{P}$, Mallamaci A, Briata P, et al. Implication of OTX2 in pigment epithelium determination and neural retina differentiation. J Neurosci. 1997; 17(11):4243-52.

13. Chen CA, Yin J, Lewis RA, et al. Genetic causes of optic nerve hypoplasia. J Med Genet. 2017;54(7):441-9. https://doi.org/10.1136/ jmedgenet-2017-104626.
14. Dateki S, Kosaka K, Hasegawa K, et al. Heterozygous orthodenticle homeobox 2 mutations are associated with variable pituitary phenotype. J Clin Endocrinol Metab. 2010;95(2):756-64. https://doi.org/ 10.1210/jc.2009-1334.

15. Bennett CP, Betts DR, Seller MJ. Deletion 14q (q22q23) associated with anophthalmia, absent pituitary, and other abnormalities. J Med Genet. 1991; 28(4):280-1.

16. Chassaing N, Causse A, Vigouroux A, et al. Molecular findings and clinical data in a cohort of 150 patients with anophthalmia/microphthalmia. Clin Genet. 2014;86(4):326-34. https://doi.org/10.1111/cge.12275.

17. North $\mathrm{KN}, \mathrm{Wu} \mathrm{BL}, \mathrm{Cao} \mathrm{BN}$, et al. CHARGE association in a child with de novo inverted duplication (14)(q22-->q24.3). Am J Med Genet. 1995;57(4):610-4. https://doi.org/10.1002/ajmg.1320570419.

\section{Ready to submit your research? Choose BMC and benefit from:}

- fast, convenient online submission

- thorough peer review by experienced researchers in your field

- rapid publication on acceptance

- support for research data, including large and complex data types

- gold Open Access which fosters wider collaboration and increased citations

- maximum visibility for your research: over $100 \mathrm{M}$ website views per year

At BMC, research is always in progress.

Learn more biomedcentral.com/submissions 